\title{
Histological Study of the Possible Therapeutic Effect of Bone Marrow Derived Mesenchymal Stem Cells on the Ultrastructure of the Corneal Keratocytes in Alkali Burn Model in Adult Male Albino Rats
}

\author{
Suzan E. Abo EInasr*, Shereen Sh. El Abd, Sadika M. El-Ebs, \\ Gehan M. Soliman and Naglaa I. Sarhan
}

Department of Histology, Faculty of Medicine, Tanta University, Egypt

*Corresponding author

\section{A B S T R A C T}

\section{Keywords}

bone marrow derived mesenchymal stem cells, Keratocytes, Chemical injuries

Article Info

Accepted:

07 October 2019

Available Online:

10 November 2019
Chemical burnsare serious ocular emergencies that require immediate interventionto minimize severe complications and visual loss. Keratocytes (corneal corpuscles) are the main cells within the corneal stroma that can be activated after injury generating myofibroblast precursors. Previous studies showed that bone marrow mesenchymal stem cells (BM-MSCs) have potential therapeutic value in corneal reconstruction. This work was performed to evaluate the ultrastructural changes of the corneal keratocytes due to alkali burn and the possible therapeutic effect of bone marrow derived mesenchymal stem cells (BM-MSCs). Thirty adult male albino rats were used and divided equally into three main groups:Group I: was the control group. Group II: alkali burn was induced and left without any treatment. Group III: alkali burn was induced and rats were treated with single subconjunctival injection of $2.4 \times 10^{6} \mathrm{BM}-\mathrm{MSC}$.In each group, half of the animals was sacrificed after one week while the other half was sacrificed after three weeks. Electron microscopic examination of corneal keratocytes of group II revealed marked alteration in the form of multiple cytoplasmic vacuoles and increase in their size in addition to nuclear indentation, condensation, bizzare shape and eccentric position. On the other hand, the stem cell treated group showed disappearance of most of these histological alternations. From the present study it could be concluded that corneal Alkali burn stimulated keratocytesto undergo cell death and to lose their quiescence state and BM-MSCs could effectively promote corneal alkali burn healing through improvement of corneal keratocytes.

\section{Introduction}

Cornea is the transparent anterior segment of the eye that refracts light onto the retina. Cornea is structured into well-organized layers, and each layer has its own importance in maintaining the viability and transparency of the tissue (Parekh et al., 2016). Keratocytes (corneal corpuscles) are the main cells within the corneal stroma and also known as fibroblasts. They are mesenchymal-derived cells and they serve to maintain the slow 
turnover of the connective tissue matrix, synthesizing collagen molecules and proteoglycans and producing matrix metalloproteinases. These cells are sparsely arranged in the stroma, yet they form an interconnected cellular network with one another through dendritic processes (Müller $e t$ al., 1995\&Hassell and Birk, 2010). Keratocytes also contain crystallins; highly expressed proteins that are known to contribute to the transparent nature of the cornea (Jester et al., 1999a).They are quiescent cells but upon injury, keratocytes are stimulated to either undergo cell death or to lose their quiescence and transition into repair phenotypes. These repair phenotypes can either promote regeneration or they can induce fibrotic scar formation which is detrimental to the transparent cornea (Fini and Stramer, 2005).

Chemical injuries to the eye represent one of the true ophthalmic emergencies. The commonest types of chemicals causing ocular injuries are acids and alkalis. Many of these are used in homes, industries and agriculture, causing burns when they come in contact with the eye, resulting in a significant threat to vision (Mashige, 2016). Ocular chemical burns are the most common cause of limbal stem cell deficiency leading to non-healing epithelial defects, stromal inflammation, neovascularization, conjunctivalization and corneal opacification (Müftüoglu et al., 2015).

Mesenchymal stem cells (MSCs) are types of multipotent cells originally isolated from bone marrow. MSCs have been applied clinically in treating a wide range of diseases for their multipotency, immunomodulatory properties, homing abilities, and the ability to secrete bioactive molecules assisting in the recovery and repair of damaged tissues. Previous studies showed that MSCs have potential therapeutic value in corneal reconstruction. Furthermore, MSCs are useful in suppressing corneal transplantation rejection and facilitating corneal wound healing (Yao and Bai, 2013 \& Chen et al., 2016).

This work was performed to demonstrate effect of BM-MSCs on corneal keratocytes after alkali burn and their role in accelerating the healing of the stroma.

\section{Materials and Methods}

\section{Study design}

This study was performed on 30 adult male albino rats. They were divided into 3 groups

\section{Control group}

It included 10 rats for ultrastructural study of the corneal keratocytes. The bone marrow also was collected from both femur and tibia of each rat and cultured for isolation of MSCs.

\section{Alkali burn induced group}

It included 10 rats. Alkali burn was induced by applying a piece of filter paper soaked in 1 $\mathrm{M} \mathrm{NaOH}$ (El-Gomhouria company for trading chemicals and medical appliances)to the center of the cornea for $30 \mathrm{sec}$. Afterwards the corneas were washed with saline for $30 \mathrm{sec}$. The burn was created on the right eye of each rat while the other eye was left without burn so that the animals could eat and move freely and then they were left without any treatment (Acar et al., 2015). Half of the animals was sacrificed after one week (subgroup IIa) while the other half was sacrificed after three weeks (subgroup II b).

\section{Stem cell treated group}

Alkali burn was induced as in group II and after 24 hours the rats were treated with bone marrow derived mesenchymal stem cells suspended in its phosphate buffered saline. 
MSCs were administrated by single subconjunctival injection in dose of $2.4 \times 10^{6}$ (Acar et al., 2015). Half of the animals was sacrificed after one week (subgroup IIIa) while the other half was sacrificed after three weeks (subgroup III b).

The animals were anesthetized by intraperitonial injection of sodium pentobarbital $(50 \mathrm{mg} / \mathrm{Kg})$ (Gaertner et al., 2008) and the eye balls were enucleated to obtain the corneas for further processing to be examined by electron microscopy.

\section{Isolation, culture of MSCs and preparation of MSC suspension}

The bone marrow of the femurs and tibias of rats were extracted, collected and cultured for isolation of mesenchymal stem cells (MSCs), it was called passage $0(\mathrm{P} 0)$. When MSCs (P0) reached confluence $(70-80 \%)$ after about (7 9) days, subculture was done to reach passage 1 (P1) which upon reaching confluence (70 $80 \%)$ trypsinization was done to reach passage 2 (P2). BM MSC of passage 2 identification was carried out by phase contrast (inverted) microscope (Zeiss-Model :Axiovert 200M Country: Germany) and immunocytochemical staining by CD44 and CD34 to recognize their characteristic features.

The rest of the cells (P2) were seeded until reaching confluence, trypsinized to get $\mathrm{P} 3$ MSCs. Then the cells were counted and injected subconjunctivallyat a dose of $2.4 \times 10^{6} /$ rat.

\section{For electron microscopy}

Small specimens of corneas $\left(1 \mathrm{~mm}^{3}\right)$ were prepared for electron microscopic examination. They were fixed in $2.5 \%$ phosphate buffered glutaraldehyde solution for $2 \mathrm{~h}$ at $4^{\circ} \mathrm{C}(\mathrm{pH} \mathrm{7.4)}$. After washing with phosphate-buffer, the specimens were post fixed in $1 \%$ buffered osmium tetroxide solution for $1 \mathrm{~h}$. After that, the specimens were dehydrated through ascending series of ethanol alcohol, treated with propylene oxide, and embedded in epoxy resin. After heat polymerization, the sections were cut using LKB ultramicrotome with a glass knife and were double-stained with uranyl acetate and lead citrate (Woods and Stirling, 2008) to be examined by a JOEL (Japan) electron microscope at the EM unit of Tanta University.

\section{Results and Discussion}

\section{Morphological identification of the primary culture of BM-MSCs}

Examination of Bone Marrow derived Mesenchymal Stem Cells (BM- MSCs) by phase contrast inverted microscope revealed suspended, crowded and rounded cells on day one of the primary culture (Fig. 1a). Some of them became adherent to the floor of the flask and spindle shaped on day three (Fig. 1b). They proliferated, assumed star shape and became maximally adherent and confluent on day twelve (Fig. 1c).

By immunohistochemical methods, BMMSCs were CD44-positive and CD34negative (Figs. 2a and 2b).

\section{Results of electron microscopy}

\section{Group I (Control group)}

Electron microscopic examination of ultra thin sections of corneal stroma of control animals displayed dispersed regularly spaced spindle shaped attenuated keratocytes with central oval nuclei. These cells demonstrated long processes and thin rim of cytoplasm. They were squeezed between the collagen lamellae (Figs. 3a and 3b). Group II (alkali burn induced group): This group showed distinct 
variation in the morphology of kertaoctytes. Subgroup IIa that was sacrificed after 1 week exhibited activated keratocytes that acquired large size with extended cytoplasmic processes and having multiple cytoplasmic vacuoles.

Their nuclei showed anisonucleosis in the form indentation, peripheral chromatin condensation, bizzare shape and eccentric position (Figs. 4, 5, 6 and 7).

After three weeks (subgroup IIb), some keratocytes showed pyknotic peripheral electron dense nuclei andindistinct disorganized cytoplasmic organelles (Fig. 8).

While in the stem cell treated group after one and three weeks, the keratocytes showed nearly normal ultrastructure similar to that of the control group. This was in the form of elongated spindle shaped cell body with central oval nuclei surrounded with regularly arranged collagen fibers (Figs. 9a and 9b).

Keratocytes (fibroblasts), are mesencyhmalderived cells of the corneal stroma. These cells are normally quiescent, but they have great plasticity to respond and transform into repair phenotypes following injury (Judith et al., 2006).Electron microscopic examination of the cornea of the control rat depicted thin regularly spaced keratocytes present among the collagen bundles. They displayed thin cytoplasm long thin cytoplasmic process with central oval single nucleus. These findings were similar to the well known histological structure of the cornea. Similarly, Song and Joo (2004) mentioned that rat cornea is composed of the epithelium, stroma with resident keratocytes, and endothelium.

In the present study, the cornea of group II (alkali burn induced group) demonstrated different phenotypes. One of these phenotypes was activated large sized keratocytes with multiple cytoplasmic vacuolation. Their nuclei acquired bizzare shape while others showed marked indentation, condensation, and eccentric position. Apoptotic keartocyteswith small pyknotic peripheral nucleus and indistinct cytoplasmic organelles were also observed. These morphological changes were in agreement with Ashby et al., (2014) who reported that the nearest keratocytes to wound underwent apoptosis and those adjacent to this area were activated to become fibroblast or myofibroblasts. These transformed cells migrated to the wound and deposit fibrotic material to fill the defect. Additionally, Lee, (2016) noted keratocyte apoptosis following corneal epithelial injury, and he explained that the damaged corneal epithelium sends soluble mediators to stromal keratocytes that cause their death via apoptosis while other keratocytes undergo transformation into fibroblasts and myofibroblasts.

Moreover, Wang et al., (2017) attributed the destruction, disorientation and loss of collagen fibers to apoptosis of keratocytes that decreased collagen production and enhancement of metalloproteinase enzyme production that degraded collagen in corneal stromal cells and increased collagen turn over with loss of stromal collagen.

Noteworthy, the corneal stroma of group III animals (BM-MSC treated group) after one and three weeks revealed reversal of these activated kertaocytes into their quiescent stageto be more or less similar to control group. Previous studies proved the role of MSCs in healing corneal burn through different mechanisms as their antiinflammatory and immunomodulatory properties. Interestingly, the present study concentrated on the role of MSCs on corneal keratocyes and their subsequent role on acceleration the wound healing. 
Fig.1 Phase contrast micrograph of a primary culture of rat BM cells, (1a) on day one of isolation and culture, shows crowded, rounded, floated and variable sized cells (thick arrow),

(1b) on day three, some cells acquire spindle shape (thick arrow) while other cells appear rounded and refractile (arrow head) and (1c) on day 12 displays an apparent increase in the confluence of the cultured cells that have multiple interdigitating processes $(\rightarrow)$ with central vesicular nuclei and multiple nucleoli (curved arrow)

(Inverted microscope, Mic. Mag. X 200)

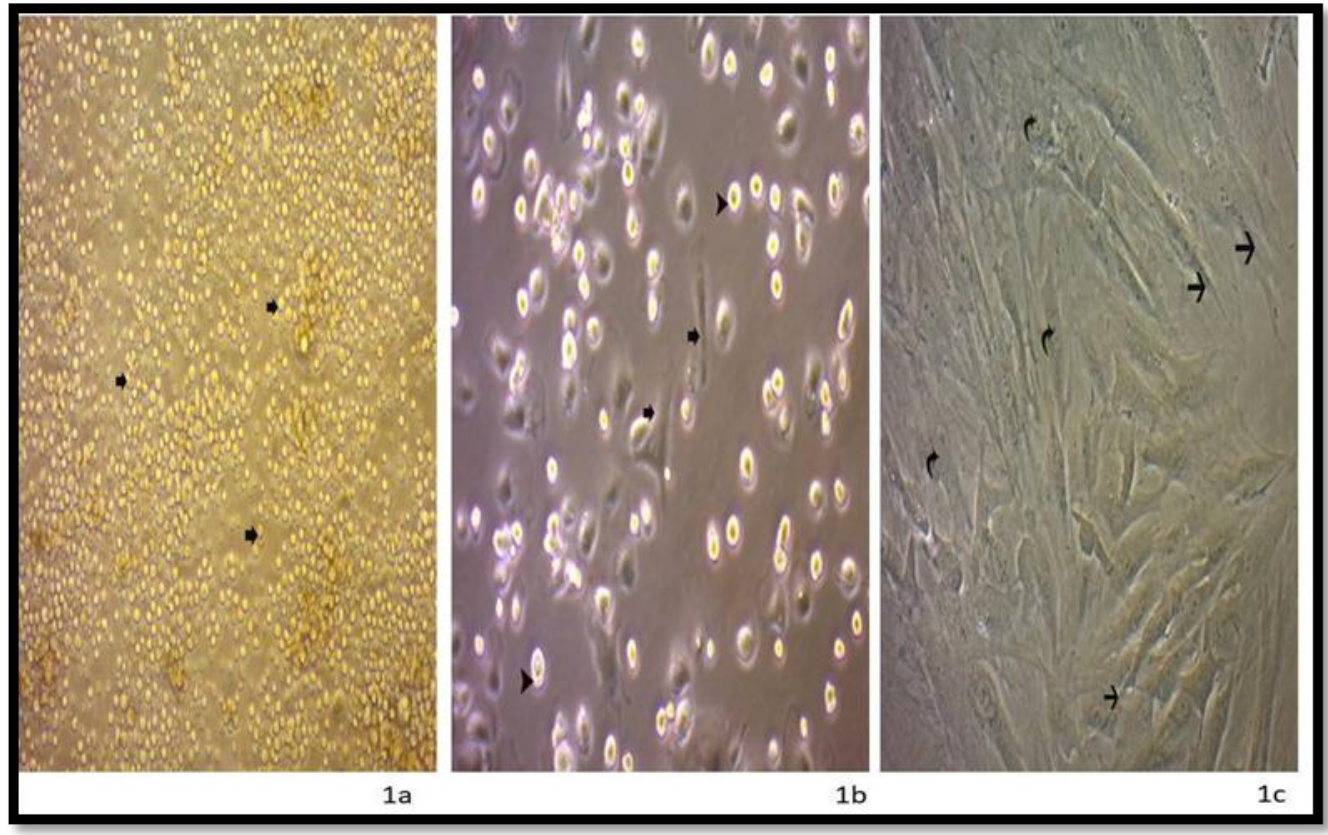

Fig.2 A Photomicrograph of primary culture of rat BM-MSCs: (2a) shows positive brownish cytoplasmic immunoreaction for CD44 (thick arrow) in most of the cells, while (2b) demonstrates totally negatively immunostained cultured cells for CD34 (thick arrow)

(CD34 Mic. Mag. X 400)

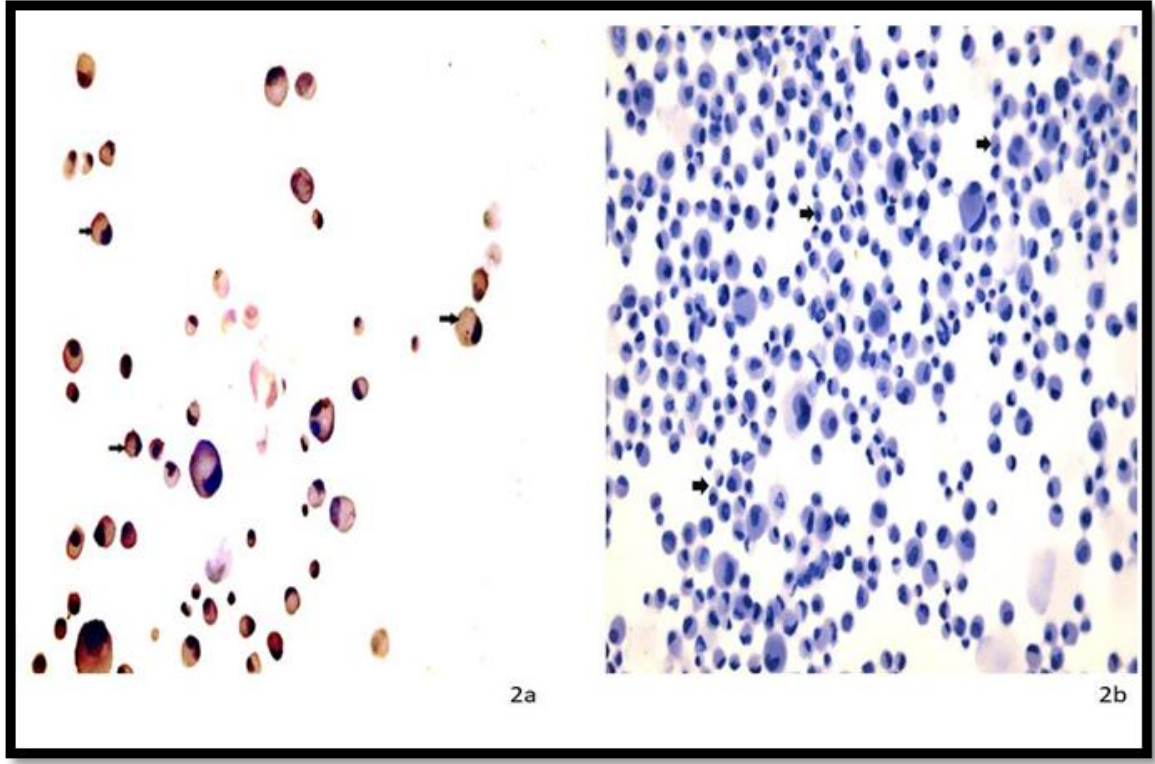


Fig.3a An electron micrograph of an ultrathin section of cornea of control group showing the long, slender attenuated keratocytes (arrow) located among the collagen bundles of the corneal stroma $(\mathrm{S})$

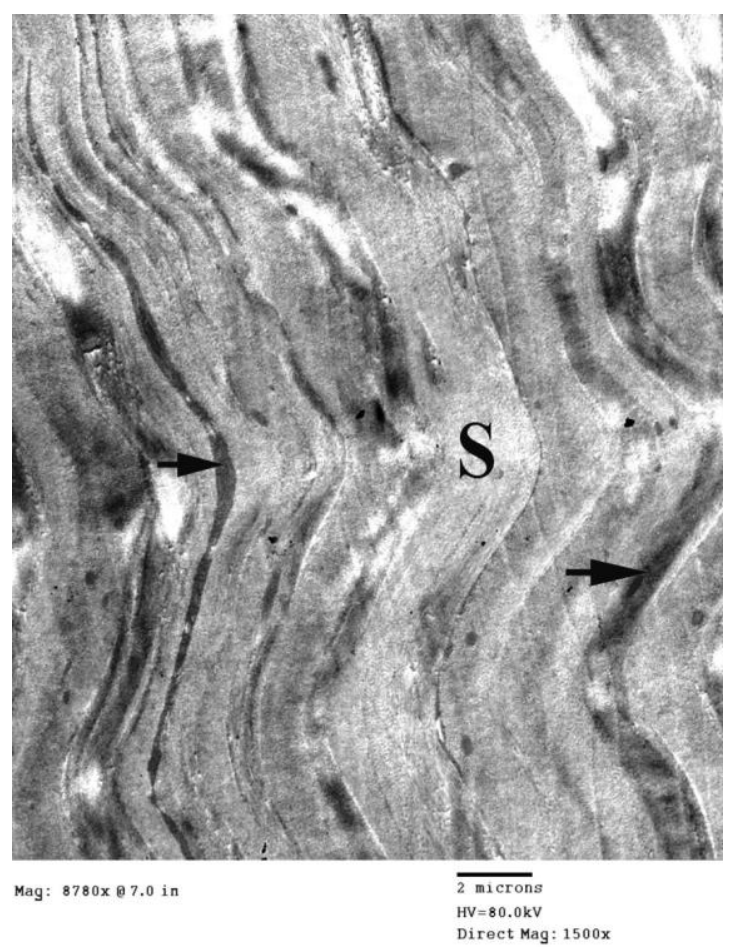

(Mic. Mag x 1500)

Fig.3b An electron micrograph of an ultrathin section of cornea of control group, showing corneal stroma (S) with thin regularly spaced keratocytes (arrow) present among the collagen fiber bundles. Note continuous non cellular Descemet's membrame (D)

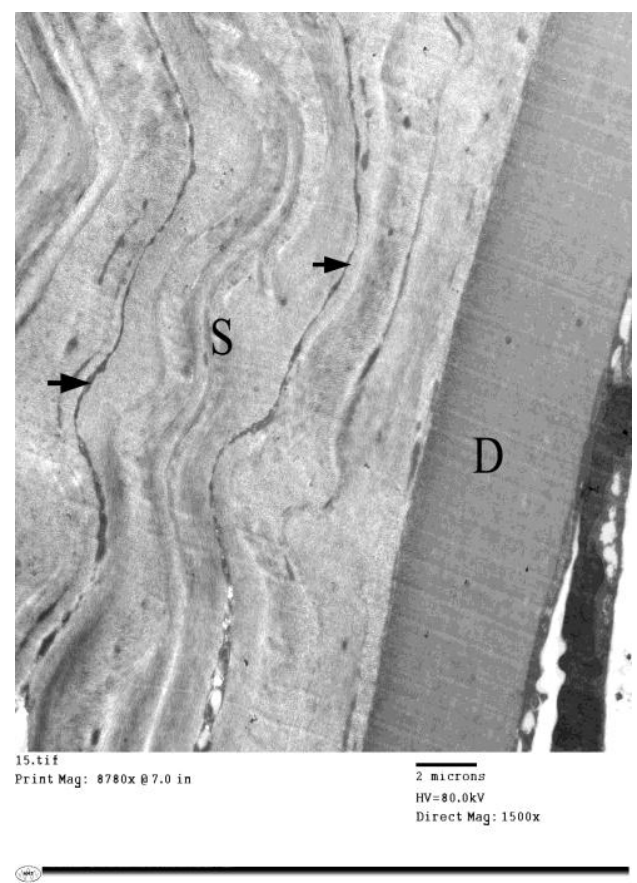

(Mic. Mag x 1500) 
Fig.4 An electron micrograph of an ultrathin section of cornea of subgroup IIa (Alkali burn induced group and sacrificed after 1 week), showing large keratocytes having eccentric, indented and condensed oval shaped nucleus (N) in association with vacuolated cytoplasm(arrow head). They are surrounded by widely displaced collagen lamellae (curved arrow) of the corneal stroma with loss of their characteristic striations (asterisk)

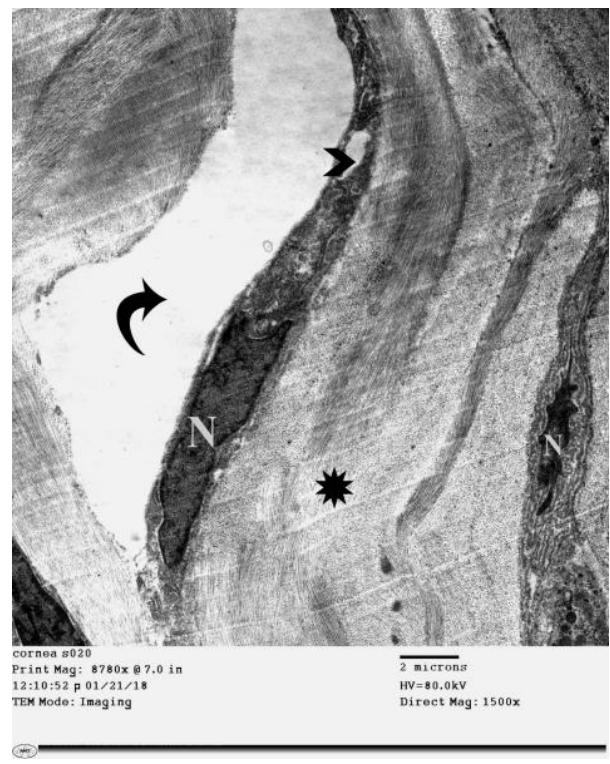

(Mic. Mag x 1500)

Fig.5 An electron micrograph of an ultrathin section of the cornea of subgroup IIa, showing activated corneal keratocytes with their large size, extended processes, multiple nuclei $(\mathrm{N})$ and cytoplasmic vacuoles (arrow head).The nuclei of these cells show peripheral chromatin condensation. Note corneal stroma (S) with focal homogenous collagen fibers losing their characteristic striations (asterisk)

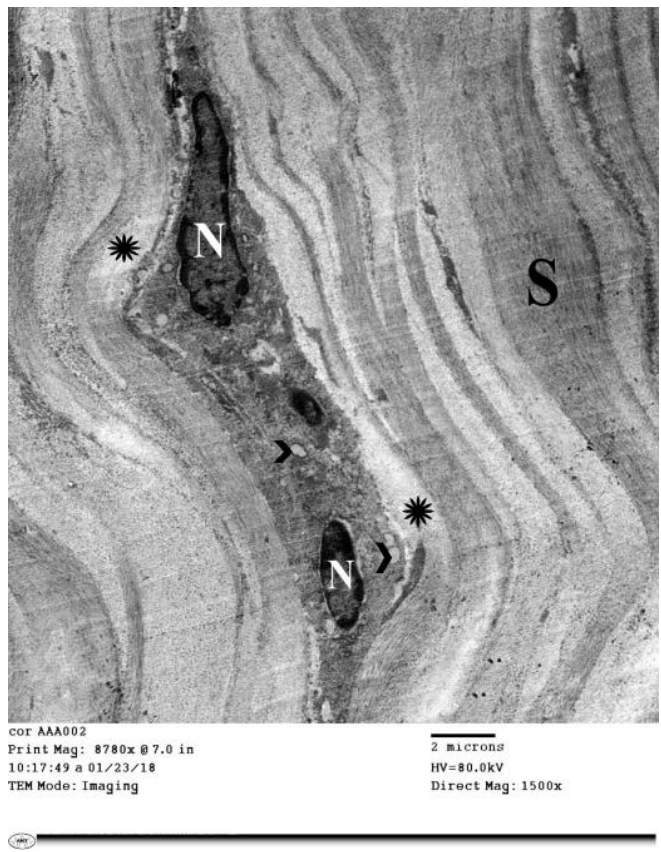

(Mic. Mag x 1500) 
Fig.6 An electron micrograph of an ultrathin section of cornea of subgroup IIa, showing altered giant keratocyte (zigzag arrow) with bizzare shaped nucleus $(\mathrm{N})$, multiple cytoplasmic vacuoles (arrow head) and extended cytoplasmic processes

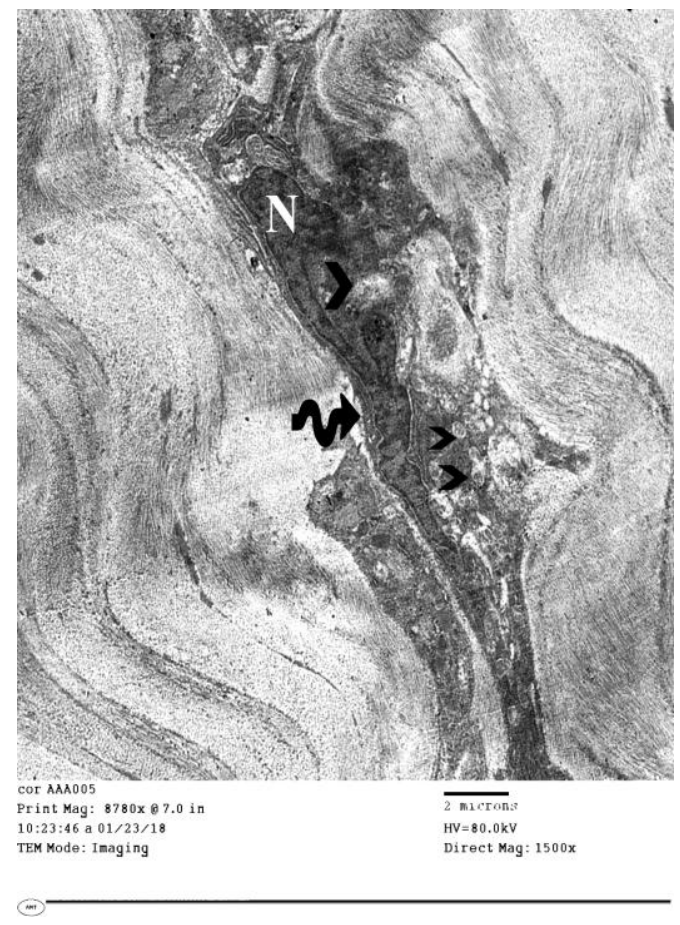

(Mic. Mag x 1500)

Fig.7 An electron micrograph of an ultrathin section of the cornea of subgroup IIa, showing swollen keratocyte (zigzag arrow) having electron dense nucleus and cytoplasmic vacuoles (arrow head) surrounded by disorganized collagen lamellae (S)

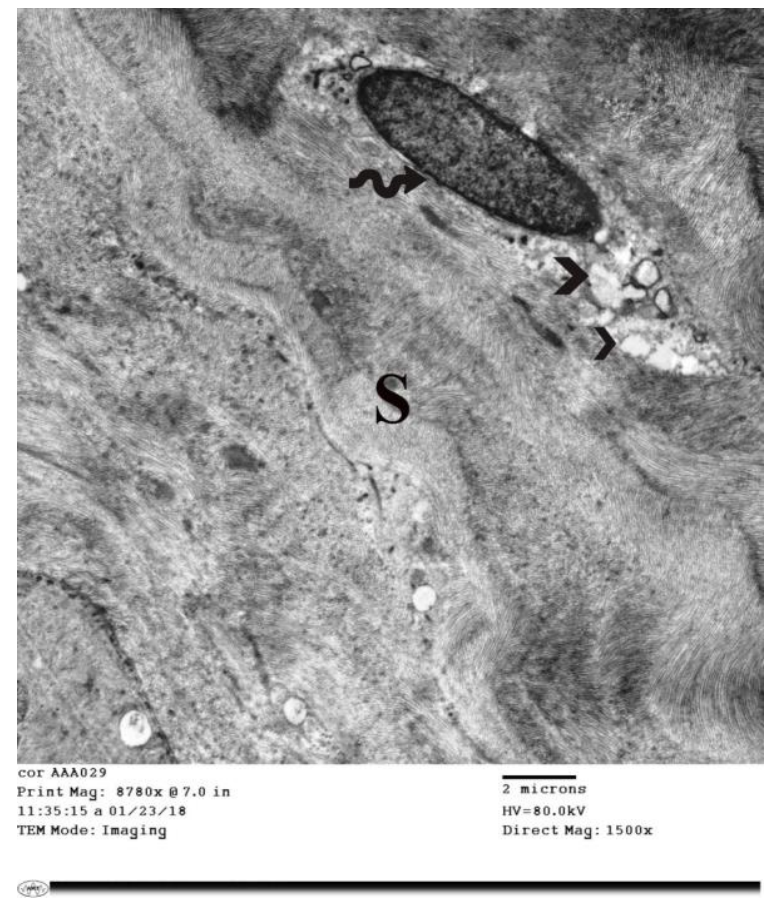

(Mic. Mag x 1500) 
Fig.8 An electron micrograph of an ultrathin section of cornea of subgroup IIb (Alkali burn induced group and sacrificed after 3 weeks), showing the apoptotic shrunken keratocyte (Zigzag arrow) with small pyknotic peripheral nucleus and indistinct cytoplasmic organelles in addition to corneal stroma (S) with focal rarified areas (star) and disorganization of the collagen fibers

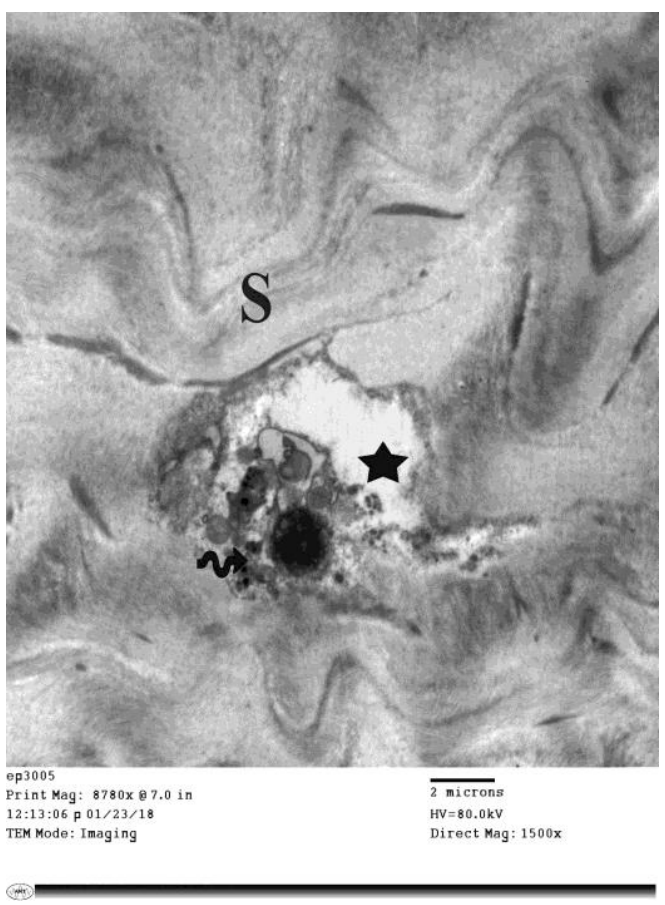

(Mic. Mag x 1500)

Fig.9 An electron micrograph of an ultrathin section of cornea of (a) subgroup IIIa (Stem cell treated group after1 week), (b) subgroup IIIb (Stem cell treated group after3 weeks) showing nearly normal keratocytes (zigzag arrow) between the regularly arranged collagen fibers of corneal stroma $(\mathrm{S})$

(Mic. Mag x 1500)
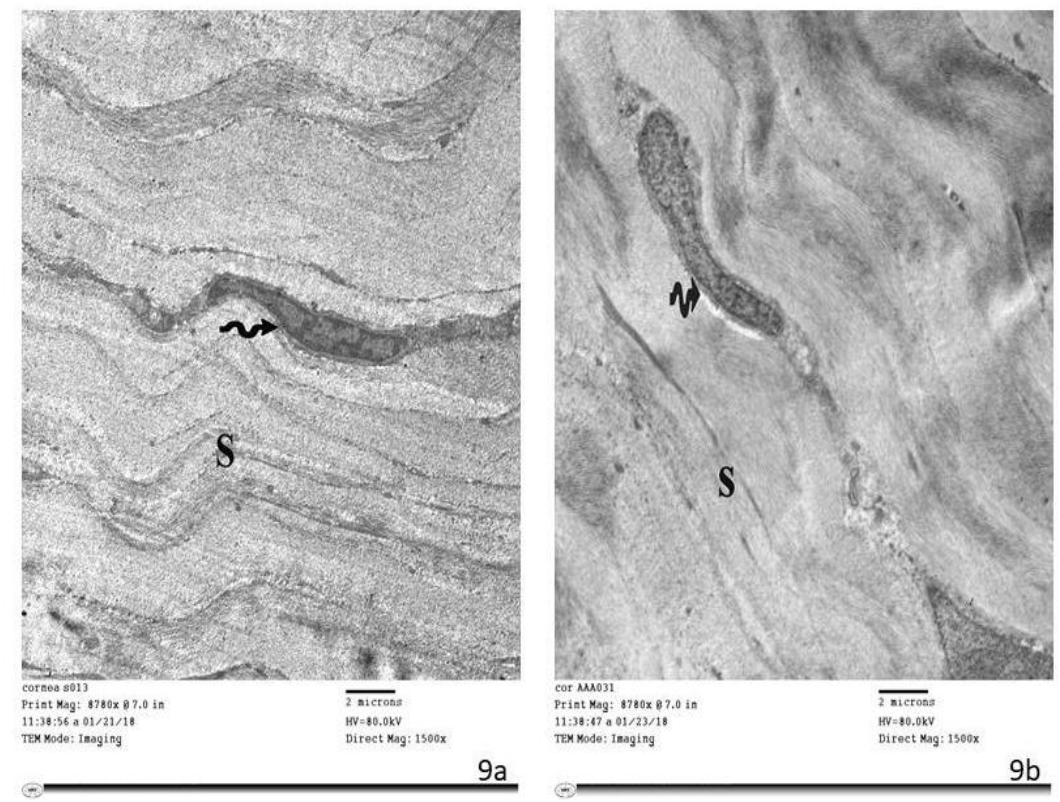
In this study, the stem cell treated group showed improvement in the ultrastructure of the corneal stroma in the form of regular arrangement of the collagen fibers of the stroma and nearly normal keratocytes between them. Zhang et al., (2015) demonstrated that BM- MSCs could be differentiated into keratocytes in animal studies. Moreover, Ebrahim et al., (2017) agreed with these findings and attributed the improvement in the corneal stroma to the regenerated keratocytes that secrete essential elements to keep the regular arrangement of the stroma, as keratansulfate proteoglycans. From this study, it could be concluded that corneal keratocytes had an important role in maintaining corneal transparency. Alkali burn causes damage to these cells and bone marrow derived mesenchymal stem cells can improve these changes.

\section{References}

Acar U, Pinarli FA, Acar DE, Beyazyildiz E, Sobaci G, Ozgermen BB. Sonmez AA and Delibasi $T$ (2015). Effect of Allogeneic Limbal Mesenchymal Stem Cell Therapy in Corneal Healing: Role of Administration Route. Ophthalmic Research; 53(2): 82-91.

Ashby BD, Garrett Q and Willcox MDP (2014): Corneal Injuries and Wound Healing - Review of Processes and Therapies, Austin Journal of Clinical Ophthalmology; 4(1): 1-25.

Chen YS, Chen YA, Tsai PH, Chen CP, Shaw SW and Hsuan Y (2016): Mesenchymal stem cell: considerations for manufacturing and clinical trials on cell therapy product. International Journal of Stem Cell Research \& Therapy; 3(1):1-12.

Ebrahim N, Mohammed OM, Dessouky AA and Abdel Fatah DS (2017): The potential therapeutic effect of stem cells loaded on two different vehicles (amniotic membrane and platelet rich plasma gel) in experimentally induced corneal alkali burns in rats. Egyptian Journal of Histology; 40(4):405-426.

Fini $\mathrm{ME}^{1}$ and Stramer BM (2005): How the cornea heals: cornea-specific repair mechanisms affecting surgical outcomes. Cornea.; 24(8 Suppl):S2S11.

Gaertner DJ, Hallman TM, Hankenson FC and Batchhelder M A (2008): Anesthesia and analgesia in rodents. In Anesthesia and analgesia in laboratory animals, Fish RE, Brown MJ, Danneman, PJ, and Karas AZ (eds), $2^{\text {nd }}$ edition, Academic Press, New York, p:239297.

Hassell JR and Birk DE (2010): The molecular basis of corneal transparency, Experimental Eye Research; (91):326-335.

Jester JV, Huang J, Barry-Lane PA, Kao WW, Petroll WM, Cavanagh HD. (1999a): Transforming growth factor (beta)mediated corneal myofibroblast differentiation requires actin and fibronectin assembly. Invest Ophthalmol Vis Sci. 40: 1959-1967.

Judith A. West-Mays and Dhruva J. Dwivedi (2006): The keratocyte: Corneal stromal cell with variable repair phenotypes. Int J Biochem Cell Biol. 2006; 38(10): 1625-1631.

Lee TN (2016): The Ins and Outs of Corneal Wound Healing, Review of Optometry: 44-54.

Mashige K (2016): Chemical and thermal ocular burns: a review of causes, clinical features and management protocol, South African Family Practice; 58(1):1-4.

Müftüoglu IK, Akova YA and Çetinkaya A (2015): Clinical Spectrum and Treatment Approaches in Corneal Burns, Turk J Ophthalmol. ; 45: 182187. 
Müller LJ ${ }^{1}$, Pels L, Vrensen GF (1995): Novel aspects of the ultrastructural organization of human corneal keratocytes. Invest Ophthalmol Vis Sci. 36(13): 2557-67.

Parekh M, Ferrari S, Sheridan C, Kaye S, and Ahmad S (2016): Concise review: An update on the culture of human corneal endothelial cells for transplantation. Stem Cells Translational Medicine; 5 (2):258-264.

Song I-K and Joo C-K (2004): Morphological and Functional Changes in the Rat Cornea with an Ethanol-Mediated Epithelial Flap. Investigative Ophthalmology \& Visual Science February 2004, (45): 423-428.

Wang M, Li M, Yan P, Luo Q, Zhang Y, and Du Z.(2017): The Effect of high intensity focused ultrasound keratoplasty on rabbit anterior segment. Journal of Ophthalmology. (2017); 1-10.

Woods A and Stirling J (2008): Electron microscope. In: Theory and practice of histological techniques, Bancroft JD and Gamble $\mathrm{M}(\mathrm{eds}), 6^{\text {th }}$ edition, Chuchill Livengstone, New York, Toronto, chapter 31; P: 601-641.

Yao L and Bai H (2013): Review: Mesenchymal stem cells and corneal reconstruction. Molecular Vision; 19: 2237-2243.

Zhang L, Coulson-Thomas VJ, Ferreira TG and Kao WWY (2015): Mesenchymal stem cells for treating ocular surface diseases. BMC Ophthalmology; 15:155.

\section{How to cite this article:}

Suzan E. Abo Elnasr, Shereen Sh. El Abd, Sadika M. El-Ebs, Gehan M. Soliman and Naglaa I. Sarhan 2019. Histological Study of the Possible Therapeutic Effect of Bone Marrow Derived Mesenchymal Stem Cells on the Ultrastructure of the Corneal Keratocytes in Alkali Burn Model in Adult Male Albino Rats. Int.J.Curr.Microbiol.App.Sci. 8(11): 545-555. doi: https://doi.org/10.20546/ijcmas.2019.811.067 